REPRESENTATION THEORY

An Electronic Journal of the American Mathematical Society

Volume 19, Pages 3-8 (March 2, 2015)

S $1088-4165(2015) 00463-7$

\title{
ON THE CHARACTER OF CERTAIN IRREDUCIBLE MODULAR REPRESENTATIONS
}

\author{
G. LUSZTIG
}

\begin{abstract}
Let $G$ be an almost simple, simply connected algebraic group over an algebraically closed field of characteristic $p>0$. In this paper we restate our conjecture from 1979 on the characters of irreducible modular representations of $G$ so that it is now directly applicable to any dominant highest weight.
\end{abstract}

1. Let $G$ be an almost simple, simply connected algebraic group over $\mathbf{k}$, an algebraically closed field of characteristic $p>1$. Let $\operatorname{Rep} G$ be the category of finite dimensional k-vector spaces with a given rational linear action of $G$ and let $\operatorname{Irr} G$ be a set of representatives for the simple objects of $\operatorname{Rep} G$. We fix a Borel subgroup $B$ of $G$ and a maximal torus $T$ of $B$. Let $Y=\operatorname{Hom}\left(\mathbf{k}^{*}, T\right), X=\operatorname{Hom}\left(T, \mathbf{k}^{*}\right)$ (with group operation written as + ) and let $\langle\rangle:, Y \times X \rightarrow \mathbf{Z}$ be the obvious pairing. If $V \in \operatorname{Irr} G$, then there is a well-defined $\lambda_{V} \in X$ with the following property: the $T$-action on the unique $B$-stable line in $V$ is through $\lambda_{V}$; according to Chevalley, $E \mapsto \lambda_{E}$ is a bijection from $\operatorname{Irr} G$ to a subset of $X$ of the form

$$
X^{+}=\left\{\lambda \in X ;\left\langle\check{\alpha}_{i}, \lambda\right\rangle \in \mathbf{N} \quad \forall i \in I\right\}
$$

for a well-defined basis $\left\{\check{\alpha}_{i} ; i \in I\right\}$ of $Y$. For $\lambda \in X^{+}$we shall denote by $V_{\lambda}$ the object of $\operatorname{Irr} G$ corresponding to $\lambda$. If $V \in \operatorname{Rep} G$, then for any $\mu \in X$ we denote by $n_{\mu}(V)$ the multiplicity of $\mu$ in $\left.V\right|_{T}$; we set $[V]=\sum_{\mu \in X} n_{\mu}(V) e^{\mu} \in \mathbf{Z}[X]$ where $\mathbf{Z}[X]$ is the group ring of $X$ (the basis element of $\mathbf{Z}[X]$ corresponding to $\mu \in X$ is denoted by $e^{\mu}$ so that $e^{\mu} e^{\mu^{\prime}}=e^{\mu+\mu^{\prime}}$ for $\left.\mu, \mu^{\prime} \in X\right)$. It is of considerable interest to compute explicitly the element $\left[V_{\lambda}\right] \in \mathbf{Z}[X]$ for any $\lambda \in X^{+}$. Let $h$ be the Coxeter number of $G$. A conjectural formula for $\left[V_{\lambda}\right]$ (assuming that $p \geq c_{G}^{0}$ where $c_{G}^{0}$ is a constant depending only on the root datum of $G$ ) was stated in L1, p. 316]. In the early 1990's it was proved (see AJS and the references there) that there exists a (necessarily unique) prime number $c_{G} \geq c_{G}^{0}$ depending only on the root datum of $G$ such that the conjectural formula in [L1, p. 316] is true if $p \geq c_{G}$ and $c_{G}$ is minimum possible (but $c_{G}$ was not explicitly determined). In $\left[\mathrm{F}\right.$, Fiebig showed that $c_{G} \leq c_{G}^{\prime}$ where $c_{G}^{\prime}$ is an explicitly known but very large constant. In Wi , Williamson, partly in collaboration with Xuhua He, showed that for infinitely many $G, c_{G}$ is much larger than $c_{G}^{0}$. Now the conjecture in [L1, p. 316] had an unsatisfactory aspect: it applied only to a finite set of $\lambda \in X^{+}$which, after application of Jantzen's results [J] on translation functors, becomes a larger but still finite set (including all $\lambda$ in $X_{\text {red }}^{+}=\left\{\lambda \in X^{+} ;\left\langle\check{\alpha}_{i}, \lambda\right\rangle \leq p-1 \quad \forall i \in I\right\}$ ); then

Received by the editors Received by the editors October 7, 2014 and, in revised form, February $2,2015$.

2010 Mathematics Subject Classification. Primary 20G99.

This work was supported in part by National Science Foundation grant DMS-1303060 and by a Simons Fellowship.

(C)2015 American Mathematical Society 
the case of a general $\lambda \in X^{+}$had to be obtained by applying the Steinberg tensor product theorem [S]. In this note I want to offer a reformulation of the conjecture in [L1, p. 316] (now a known theorem for $p$ large enough) which applies directly to any $\lambda \in X^{+}$; see $7(\mathrm{~b})$.

2. Notation. Let $N T$ be the normalizer of $T$ in $G$ and let $W=N T / T$ be the Weyl group. Note that $W$ acts naturally on $T$ hence on $Y, X$ and $\mathbf{Z}[X]$. Let $\check{R}=\left\{y \in Y ; y=w\left(\check{\alpha}_{i}\right)\right.$ for some $\left.w \in W, i \in I\right\}$ (the set of coroots). Define $\check{\alpha}_{0} \in \check{R}$ by the condition that $\check{\alpha}_{0}+\check{\alpha}_{i} \notin \check{R}$ for any $i \in I$. (Thus $\check{\alpha}_{0}$ is the highest coroot.) For $i \in I \sqcup\{0\}$ define $\alpha_{i} \in X$ by the condition that the map $X \rightarrow X$, $\lambda \mapsto \lambda-\left\langle\check{\alpha}_{i}, \lambda\right\rangle \alpha_{i}$, is induced by a (uniquely defined) element $s_{i}$ of $W$. Let $w \mapsto \epsilon_{w}$ be the homomorphism $W \rightarrow\{1,-1\}$ such that $\epsilon_{s_{i}}=-1$ for any $i \in I$. Define $\rho \in X^{+}$by $\left\langle\check{\alpha}_{i}, \rho\right\rangle=1$ for any $i \in I$. Let $\leq$ be the partial order on $X$ given by $\lambda \leq \lambda^{\prime}$ whenever $\lambda^{\prime}-\lambda \in \sum_{i \in I} \mathbf{N} \alpha_{i}$.

3. Let

$$
\delta=\left\{\lambda \in X ;\left\langle\check{\alpha}_{i}, \lambda+\rho\right\rangle \leq 0 \quad \forall i \in I,\left\langle\check{\alpha}_{0}, \lambda+\rho\right\rangle \geq-p\right\} .
$$

For $i \in I$ we define $s_{i}^{\prime}: X \rightarrow X$ by $s_{i}^{\prime}(\lambda)=\lambda-\left\langle\check{\alpha}_{i}, \lambda+\rho\right\rangle \alpha_{i}$ (an affine reflection). We define $s_{0}^{\prime}: X \rightarrow X$ by $s_{0}^{\prime}(\lambda)=\lambda-\left(\left\langle\check{\alpha}_{0}, \lambda+\rho\right\rangle+p\right) \alpha_{0}$ (an affine reflection). Let $W_{a}$ be the subgroup of the group of permutations of $X$ generated by $s_{i}^{\prime}(i \in I \cup\{0\})$. Then $W_{a}$ is a Coxeter group on the generators $s_{i}^{\prime}(i \in I \cup\{0\})$, with length function $l: W_{a} \rightarrow \mathbf{N}$.

For $\lambda \in X$ we have $w^{-1}(\lambda) \in \delta$ for some $w \in W_{a}$ and among all such $w$ there is a unique one, $w_{\lambda}$, of minimal length.

For $\lambda, \mu \in X^{+}$we set $w=w_{\lambda}$ and

$$
\mathbf{p}_{\mu, \lambda}=\sum_{y \in W_{a} ; y^{-1}(\mu)=w^{-1}(\lambda)}(-1)^{l(y w)} P_{y, w}(1) \in \mathbf{Z}
$$

where $P_{y, w}$ is the polynomial associated in [KL] to $y, w$ in the Coxeter group $W_{a}$.

From the definitions we see that $\mathbf{p}_{\mu, \lambda} \neq 0 \Longrightarrow \mu \leq \lambda, \mathbf{p}_{\lambda, \lambda}=1$. Hence for $\lambda, \mu \in X^{+}$we can define $\mathbf{q}_{\mu, \lambda} \in \mathbf{Z}$ by the requirement

$$
\sum_{\nu \in X^{+}} \mathbf{p}_{\mu, \nu} \mathbf{q}_{\nu, \lambda}=\delta_{\mu, \lambda}
$$

for any $\lambda, \mu$ in $X^{+}$. We have $\mathbf{q}_{\mu, \lambda} \neq 0 \Longrightarrow \mu \leq \lambda$ and $\mathbf{q}_{\lambda, \lambda}=1$.

4. For any $\lambda \in X^{+}$we can write uniquely $\lambda=\sum_{k \geq 0} p^{k} \lambda^{k}$ where $\lambda^{k} \in X_{\text {red }}^{+}$for all $k \geq 0$ and $\lambda^{k}=0$ for large $k$.

For any $\lambda \in X^{+}$and any $k \in \mathbf{N}$ we define elements $E_{\lambda}^{k} \in \mathbf{Z}[X]$ by induction on $k$ as follows:

$$
E_{\lambda}^{0}=\sum_{w \in W} \epsilon_{w} e^{w(\lambda+\rho)} / \sum_{w \in W} \epsilon_{w} e^{w(\rho)},
$$

$$
E_{\lambda}^{k}=\sum_{\mu \in X^{+}} \mathbf{p}_{\mu, \sum_{j ; j \geq k-1} p^{j-k+1} \lambda^{j}} E_{\sum_{j ; 0}^{k-1}{ }_{j \leq k-2} p^{j} \lambda^{j}+p^{k-1} \mu} \text { if } k \geq 1 .
$$

Note that $E_{\lambda}^{k} \in \mathbf{Z}[X]^{W}$, the ring of $W$-invariants in $\mathbf{Z}[X]$.

We show that for $k \geq 0$ we have

$$
E_{\lambda}^{k}=\sum_{\mu \in X^{+}} \mathbf{q}_{\mu, \sum_{j ; j \geq k} p^{j-k} \lambda^{j}} E_{\sum_{j ; 0 \leq j \leq k-1}^{k+1} p^{j} \lambda^{j}+p^{k} \mu} .
$$


For any $\mu \in X^{+}$and any $k, h \geq 0$,

$$
\left(\sum_{j ; 0 \leq j \leq k-1} p^{j} \lambda^{j}+p^{k} \mu\right)^{h}
$$

is equal to $\lambda^{h}$ if $0 \leq h \leq k-1$ and to $\mu^{h-k}$ if $h \geq k$; hence, by (a), we have

$$
E_{\sum_{j ; 0 \leq j \leq k-1}^{k+1} p^{j} \lambda^{j}+p^{k} \mu}=\sum_{\nu \in X^{+}} \mathbf{p}_{\nu, \sum_{h ; h \geq k} p^{h-k} \mu^{h-k}} E_{\sum_{h ; 0 \leq h \leq k-1}^{k} p^{h} \lambda^{h}+p^{k} \nu} .
$$

Thus the right-hand side of (b) is

$$
\begin{aligned}
& \sum_{\mu \in X^{+}} \mathbf{q}_{\mu, \sum_{j ; j \geq k} p^{j-k} \lambda^{j}} \sum_{\nu \in X^{+}} \mathbf{p}_{\nu, \sum_{h ; h \geq k} p^{h-k} \mu^{h-k}} E_{\sum_{h ; 0 \leq h \leq k-1}^{k} p^{h} \lambda^{h}+p^{k} \nu} \\
& =\sum_{\mu \in X^{+}} \mathbf{q}_{\mu, \sum_{j ; j \geq k} p^{j-k} \lambda^{j}} \sum_{\nu \in X^{+}} \mathbf{p}_{\nu, \mu} E_{\sum_{h ; 0 \leq h \leq k-1}^{k} p^{h} \lambda^{h}+p^{k} \nu} \\
& =\sum_{\nu \in X^{+}} \delta_{\nu, \sum_{j ; j \geq k}} p^{j-k} \lambda^{j} \\
& =E_{\sum_{h ; 0 \leq h \leq k-1}^{k}}^{k} p^{h} \lambda^{h}+p^{k} \nu \\
& =E_{\lambda ; 0 \leq h \leq k-1}^{k}
\end{aligned}
$$

This proves (b).

By induction on $k$ we see, using (a), that for any $k \geq 1$ we have

$$
\begin{aligned}
E_{\lambda}^{k}= & \sum_{\mu_{0}, \mu_{1}, \ldots, \mu_{k-1} \text { in } X^{+}} \mathbf{p}_{\mu_{0}, \lambda^{0}+p \mu_{1}} \mathbf{p}_{\mu_{1}, \lambda^{1}+p \mu_{2}} \ldots \mathbf{p}_{\mu_{k-2}, \lambda^{k-2}+p \mu_{k-1}} \\
& \times \mathbf{p}_{\mu_{k-1}, \sum_{j \geq k-1} p^{j-k+1} \lambda^{j}} E_{\mu_{0}}^{0} .
\end{aligned}
$$

By induction on $k$ we see, using (b), that for any $k \geq 1$ we have

$$
\begin{aligned}
E_{\lambda}^{0}= & \sum_{\nu_{0}, \nu_{1}, \ldots, \nu_{k-1} \text { in } X^{+}} \mathbf{q}_{\nu_{0}, \lambda} \mathbf{q}_{\nu_{1},\left(\nu_{0}-\nu_{0}^{0}\right) / p} \mathbf{q}_{\nu_{2},\left(\nu_{1}-\nu_{1}^{0}\right) / p} \cdots \mathbf{q}_{\nu_{k-1},\left(\nu_{k-2}-\nu_{k-2}^{0}\right) / p} \\
& \times E_{\nu_{0}^{0}+p \nu_{1}^{0}+\cdots+p^{k-2} \nu_{k-2}^{0}+p^{i-1} \nu_{k-1}}^{k} .
\end{aligned}
$$

5. Let $\lambda \in X^{+}$. We can find $n \geq 0$ such that $\lambda^{n}=\lambda^{n+1}=\cdots=0$. If $k \geq n$ we have

$$
E_{\lambda}^{k}=E_{\lambda}^{k+1}
$$

Indeed, if $\mu \in X^{+}$and $\mathbf{q}_{\mu, \sum_{j ; j \geq k} p^{j-k} \lambda^{j}} \neq 0$ we have $\mathbf{q}_{\mu, 0} \neq 0$ hence $\mu \leq 0, \mu=0$ and $\mathbf{q}_{0,0}=1$; it follows that

$$
E_{\lambda}^{k}=E_{\sum_{j ; 0 \leq j \leq k-1}^{k+1} p^{j} \lambda^{j}}^{k+1}=E_{\lambda}^{k+1} .
$$

Thus we can set $E_{\lambda}^{\infty}=E_{\lambda}^{k}$ for large $k$. Clearly, if $\lambda \in X_{\text {red }}^{+}$, then $E_{\lambda}^{1}=E_{\lambda}^{2}=\cdots=$ $E_{\lambda}^{\infty}$.

Letting $k \rightarrow \infty$ in $4(\mathrm{c}),(\mathrm{d})$, we deduce that

$$
E_{\lambda}^{\infty}=\sum_{\mu_{0}, \mu_{1}, \mu_{2}, \ldots \text { in } X^{+} ; \mu_{h}=0 \text { for large } h}\left(\mathbf{p}_{\mu_{0}, \lambda^{0}+p \mu_{1}} \mathbf{p}_{\mu_{1}, \lambda^{1}+p \mu_{2}} \mathbf{p}_{\mu_{2}, \lambda^{2}+p \mu_{3}} \ldots\right) E_{\mu_{0}}^{0},
$$


(note that for large $h$ we have $\mathbf{p}_{\mu_{h}, \lambda^{h}+p \mu_{h+1}}=\mathbf{p}_{0,0}=1$ so that the infinite product makes sense) and

$$
E_{\lambda}^{0}=\sum_{\nu_{0}, \nu_{1}, \nu_{2}, \ldots \text { in } X^{+}}\left(\mathbf{q}_{\nu_{0}, \lambda} \mathbf{q}_{\nu_{1},\left(\nu_{0}-\nu_{0}^{0}\right) / p} \mathbf{q}_{\nu_{2},\left(\nu_{1}-\nu_{1}^{0}\right) / p} \ldots\right) E_{\nu_{0}^{0}+p \nu_{1}^{0}+p^{2} \nu_{2}^{0}+\ldots}^{\infty}
$$

(note that for large $h$ we have $\nu_{h}=0$ hence $\mathbf{q}_{\nu_{h+1},\left(\nu_{h}-\nu_{h}^{0}\right) / p}=\mathbf{q}_{0,0}=1$ so that the infinite product makes sense).

6. It is known since the early 1990's that, if $p$ is not very small, then the conjecture 8.2 in [L2] on quantum groups at a $p$-th root of 1 holds. In particular for $\lambda \in$ $X^{+}$, the element $E_{\lambda}^{1}$ describes the character of an irreducible finite dimensional representation of such a quantum group and the tensor product theorem [L2, 7.4] holds for it.

Thus, if for any $\xi=\sum_{\lambda \in X} c_{\lambda} e^{\lambda} \in \mathbf{Z}[X]$ (with $c_{\lambda} \in \mathbf{Z}$ ) and any $h \geq 0$ we set $\xi^{(h)}=\sum_{\lambda \in X} c_{\lambda} e^{p^{h} \lambda} \in \mathbf{Z}[X]$, then for any $\lambda \in X^{+}$we have the equality

$$
E_{\lambda}^{1}=E_{\lambda^{0}}^{1}\left(E_{\sum_{j \geq 1} p^{j-1} \lambda^{j}}^{0}\right)^{(1)} .
$$

This can be also deduced from results in $[\mathrm{K}$.

We show by induction on $k \geq 1$ that for any $\lambda \in X^{+}$we have

$$
E_{\lambda}^{k}=E_{\lambda^{0}}^{1}\left(E_{\lambda^{1}}^{1}\right)^{(1)} \ldots\left(E_{\lambda^{k-1}}^{1}\right)^{(k-1)}\left(E_{\sum_{j \geq k}^{0} p^{j-k} \lambda^{j}}\right)^{(k)} .
$$

By (a), we can assume that $k \geq 2$. Using 4(a), it is enough to show that

$$
\begin{aligned}
& \sum_{\mu \in X^{+}} \mathbf{p}_{\mu, \sum_{j ; j \geq k-1} p^{j-k+1} \lambda^{j}} E_{\sum_{j ; 0 \leq j \leq k-2}^{k-1}}^{k-p^{j} \lambda^{j}+p^{k-1} \mu} \\
& \quad=E_{\lambda^{0}}^{1}\left(E_{\lambda^{1}}^{1}\right)^{(1)} \ldots\left(E_{\lambda^{k-1}}^{1}\right)^{(k-1)}\left(E_{\sum_{j \geq k}^{0} p^{j-k} \lambda^{j}}\right)^{(k)} .
\end{aligned}
$$

Replacing here

$$
E_{\sum_{j ; 0 \leq j \leq k-2} p^{j} \lambda^{j}+p^{k-1} \mu}^{k-1}=E_{\lambda^{0}}^{1}\left(E_{\lambda^{1}}^{1}\right)^{(1)} \ldots\left(E_{\lambda^{k-2}}^{1}\right)^{(k-2)}\left(E_{\mu}^{0}\right)^{(k-1)},
$$

which is known from the induction hypothesis, we see that it is enough to show that

$$
\begin{gathered}
\sum_{\mu \in X^{+}} \mathbf{p}_{\mu, \sum_{j ; j \geq k-1} p^{j-k+1} \lambda^{j}} E_{\lambda^{0}}^{1}\left(E_{\lambda^{1}}^{1}\right)^{(1)} \ldots\left(E_{\lambda^{k-2}}^{1}\right)^{(k-2)}\left(E_{\mu}^{0}\right)^{(k-1)} \\
=E_{\lambda^{0}}^{1}\left(E_{\lambda^{1}}^{1}\right)^{(1)} \ldots\left(E_{\lambda^{k-1}}^{1}\right)^{(k-1)}\left(E_{\sum_{j \geq k}}^{0} p^{j-k} \lambda^{j}\right)^{(k)} .
\end{gathered}
$$

Thus, it is enough to show that

$$
\sum_{\mu \in X^{+}} \mathbf{p}_{\mu, \sum_{j ; j \geq k-1} p^{j-k+1} \lambda^{j}}\left(E_{\mu}^{0}\right)^{(k-1)}=\left(E_{\lambda^{k-1}}^{1}\right)^{(k-1)}\left(E_{\sum_{j \geq k}^{0} p^{j-k} \lambda^{j}}\right)^{(k)}
$$

or that

$$
\sum_{\mu \in X^{+}} \mathbf{p}_{\mu, \sum_{j ; j \geq k-1} p^{j-k+1} \lambda^{j}} E_{\mu}^{0}=E_{\lambda^{k-1}}^{1}\left(E_{\sum_{j \geq k}^{0} p^{j-k} \lambda^{j}}\right)^{(1)} .
$$

Using (a), the right-hand side is $E_{\sum_{j \geq k-1} p^{j-k+1} \lambda^{j}}$. This is equal to the left-hand side, by $4(\mathrm{a})$. This proves (b).

Letting $k \rightarrow \infty$ in (b) we obtain for any $\lambda \in X^{+}$:

$$
E_{\lambda}^{\infty}=E_{\lambda^{0}}^{1}\left(E_{\lambda^{1}}^{1}\right)^{(1)}\left(E_{\lambda^{2}}^{1}\right)^{(2)} \ldots
$$


Note that for large $h$ we have $\lambda^{h}=0$ hence $E_{\lambda^{h}}^{1}=1$ so that the infinite product makes sense. (We have also used that $E_{\sum_{j \geq k} p^{j-k} \lambda^{j}}^{0}=E_{0}^{0}=1$ for large $h$.)

7. We now assume that $p \geq c_{G}$. Then, by the first paragraph of section 6 , we have

$$
\left[V_{\lambda}\right]=E_{\lambda}^{1} \text { for any } \lambda \in X_{\text {red }}^{+} \text {. }
$$

Using the Steinberg tensor product theorem $[\underline{S}]$ and (a), we see that for any $\lambda \in X^{+}$ we have

$$
\left[V_{\lambda}\right]=\left[V_{\lambda^{0}}\right]\left[V_{\lambda^{1}}\right]^{(1)}\left[V_{\lambda^{2}}\right]^{(2)}=\cdots=E_{\lambda^{0}}^{1}\left(E_{\lambda^{1}}^{1}\right)^{(1)}\left(E_{\lambda^{2}}^{1}\right)^{(2)} \cdots
$$

Using this and $6(\mathrm{c})$ we deduce

$$
\left[V_{\lambda}\right]=E_{\lambda}^{\infty} .
$$

8. We preserve the setup of section 7 . In the identity

$$
E_{\lambda}^{0}=\sum_{\mu \in X^{+}} \mathbf{q}_{\mu, \lambda} E_{\mu}^{1}
$$

(see 4(b)) the coefficient $\mathbf{q}_{\mu, \lambda}$ can be interpreted as the multiplicity of an irreducible representation of a quantum group at a $p$-th root of 1 in a not necessarily irreducible representation of that quantum group. In particular we have

$$
\mathbf{q}_{\mu, \lambda} \in \mathbf{N}
$$

for any $\lambda, \mu$ in $X^{+}$. We show by descending induction on $k$ that for any $\lambda \in X^{+}$ and any $k \geq 0$ we have

$$
E_{\lambda}^{k}=\left[V_{\lambda}(k)\right]
$$

for some $V_{\lambda}(k) \in \operatorname{Rep} G$.

If $k$ is large, we have $E_{\lambda}^{k}=E_{\lambda}^{\infty}$ and (b) follows from 7(b). Assume now that $k \geq 0$ and that (b) is known when $k$ is replaced by $k+1$. Then (b) holds for $k$ by (a), 4(b) and the induction hypothesis.

9. We now assume that $G=S L_{2}(\mathbf{k})$ and $p=3$. Let $d_{\lambda}(k)=\operatorname{dim} V_{\lambda}(k)$ (see Sec. 8) where $\lambda \in X^{+}$and $k \geq 0$. The numbers $d_{\lambda}(0)$ form a sequence

$$
\begin{array}{lllllllllllllllllll}
1 & 2 & 3 & 4 & 5 & 6 & 7 & 8 & 9 & 10 & 11 & 12 & 13 & 14 & 15 & 16 & 17 & 18 & 19 \ldots
\end{array}
$$

where $X^{+}$is identified with $\mathbf{N}$ in an obvious way. We underline the numbers divisible by 3 in this sequence:

$$
\begin{array}{lllllllllllllllllll}
1 & 2 & \underline{3} & 4 & 5 & \underline{6} & 7 & 8 & \underline{9} & 10 & 11 & \underline{12} & 13 & 14 & \underline{15} & 16 & 17 & \underline{18} & 19 \ldots
\end{array}
$$

and regard the underlined numbers as mirrors. We now consider the reflections of the non-mirrors in the list in the mirrors to its left and replace them by the alternating sum of those reflections; we leave the mirrors unchanged. For example, 4 is replaced by $4-2=2,5$ is replaced by $5-1=4,7$ is replaced by $7-5+1=3$, 8 is replaced by $8-4+2=6$, etc. We obtain the sequence

$$
\begin{array}{lllllllllllllllllll}
1 & 2 & 3 & 2 & 4 & 6 & 3 & 6 & 9 & 4 & 8 & 12 & 5 & 10 & 15 & 6 & 12 & 18 & 7 \ldots
\end{array}
$$

which is the same as the sequence $d_{\lambda}(1)$. We arrange the numbers in the last sequence in groups of three and we underline every third group (in which all numbers are divisible by 3$)$ :
$[1$
2 3] $[2 \quad 4$
$4 \quad 6] \quad \underline{\left[\begin{array}{lll}3 & 6 & 9\end{array}\right]}$
$\left[\begin{array}{lll}4 & 8 & 12\end{array}\right]$
$\left[\begin{array}{ll}5 & 10\end{array}\right.$
15] $\underline{\left[\begin{array}{lll}6 & 12 & 18\end{array}\right]}[7 \ldots]$. 
We regard the underlined groups as (new) mirrors. We consider the reflections of the non-(new) mirrors in the list in the (new) mirrors to its left (preserving the order in each group) and replace them by the alternating sum of those reflections; we leave the (new) mirrors unchanged. For example, $\left[\begin{array}{lll}4 & 8 & 12\end{array}\right]$ is replaced by $\left[\begin{array}{lll}4 & 8 & 12\end{array}\right]-\left[\begin{array}{lll}2 & 4 & 6\end{array}\right]=\left[\begin{array}{lll}2 & 4 & 6\end{array}\right],\left[\begin{array}{lll}5 & 10 & 15\end{array}\right]$ is replaced by $\left[\begin{array}{lll}5 & 10 & 15\end{array}\right]-$ $\left[\begin{array}{lll}1 & 2 & 3\end{array}\right]=\left[\begin{array}{lll}4 & 8 & 12\end{array}\right],\left[\begin{array}{lll}7 & 14 & 21\end{array}\right]$ is replaced by $\left[\begin{array}{lll}7 & 14 & 21\end{array}\right]-\left[\begin{array}{lll}5 & 10 & 15\end{array}\right]+$ $\left[\begin{array}{lll}1 & 2 & 3\end{array}\right]=\left[\begin{array}{lll}3 & 6 & 9\end{array}\right]$, etc. We obtain the sequence

$$
\begin{array}{lllllllllllllllllllll}
1 & 2 & 3 & 2 & 4 & 6 & 3 & 6 & 9 & 2 & 4 & 6 & 4 & 8 & 12 & 6 & 12 & 18 & 3 \ldots
\end{array}
$$

which is the same as the sequence $d_{\lambda}(2)$. We now arrange the numbers in the last sequence in groups of nine and we underline every third group (in which all numbers are divisible by 3 ). We repeat the previous procedure and we get the sequence $d_{\lambda}(3)$, then $d_{\lambda}(4)$, etc.

\section{REFERENCES}

[AJS] H. H. Andersen, J. C. Jantzen, and W. Soergel, Representations of quantum groups at a pth root of unity and of semisimple groups in characteristic $p$ : independence of $p$, Astérisque 220 (1994), 321. MR 1272539 (95j:20036)

[F] P. Fiebig, An upper bound on the exceptional characteristics for Lusztig's character formula, J. Reine Angew. Math. 673 (2012), 1-31, DOI 10.1515/CRELLE.2011.170. MR2999126

[J] J. C. Jantzen, Moduln mit einem höchsten Gewicht (German), Lecture Notes in Mathematics, vol. 750, Springer, Berlin, 1979. MR552943 (81m:17011)

[K] S.-i. Kato, On the Kazhdan-Lusztig polynomials for affine Weyl groups, Adv. in Math. 55 (1985), no. 2, 103-130, DOI 10.1016/0001-8708(85)90017-9. MR772611 (86d:20048)

[KL] D. Kazhdan and G. Lusztig, Representations of Coxeter groups and Hecke algebras, Invent. Math. 53 (1979), no. 2, 165-184, DOI 10.1007/BF01390031. MR.560412 (81j:20066)

[L1] G. Lusztig, Some problems in the representation theory of finite Chevalley groups, The Santa Cruz Conference on Finite Groups (Univ. California, Santa Cruz, Calif., 1979), Proc. Sympos. Pure Math., vol. 37, Amer. Math. Soc., Providence, RI, 1980, pp. 313-317. MR604598 (82i:20014)

[L2] G. Lusztig, Modular representations and quantum groups, Classical groups and related topics (Beijing, 1987), Contemp. Math., vol. 82, Amer. Math. Soc., Providence, RI, 1989, pp. 59-77, DOI 10.1090/conm/082/982278. MR982278 (90a:16008)

[S] R. Steinberg, Representations of algebraic groups, Nagoya Math. J. 22 (1963), 33-56. MR0155937(27 \#5870)

[Wi] G.Williamson, Schubert calculus and torsion, arxiv:1309.5055.

Department of Mathematics, M.I.T., Cambridge, Massachusetts 02139

E-mail address: gyuri@math.mit.edu 